\title{
ACYL-COENZYME A FORMATION OF SIMVASTATIN IN MOUSE LIVER PREPARATIONS
}

Chunze Li, Raju Subramanian ${ }^{1}$, Sean Yu and Thomayant Prueksaritanont

Department of Drug Metabolism, Merck Research Laboratories, West Point,

Pennsylvania (C. L., R.S., S.Y., T. P.) 


\section{DMD \# 6650}

Running title: Acyl-CoA Formation of Simvastatin in Mice

Corresponding author: Dr. Chunze Li, Department of Drug Metabolism, Merck

Research Laboratories, WP 75-100, P. O. Box 4, West Point, PA 19486. Email:

chunze_li@merck.com

Number of text pages: 22

Number of tables: 3

Number of figures: 7

Number of References: 30

Number of words in Abstract: 230

Number of words in Introduction: 410

Number of words in Discussion: 1494

Abbreviations used are: HMG-CoA, 3-hydroxy-3-methylglutaryl-coenzyme A; SVA, hydroxy acid form of simvastatin; LVA, hydroxy acid form of lovastatin; SVA-CoA, SVA-S-acyl coenzyme A; P1-CoA, $S$-acyl coenzyme A of P1 metabolite; P2-CoA, $S$-acyl coenzyme A of P2 metabolite; PA-CoA, palmitoyl-S-acyl coenzyme A; OA-CoA, octanoyl-S-acyl coenzyme A; HPLC, high-pressure liquid chromatography; LC-MS, liquid chromatography-mass spectrometry; MS/MS, tandem mass spectrometry; Q-TOF, quadrupole time-of-flight. 


\title{
DMD \# 6650
}

\author{
$\underline{\text { Abstract: }}$ \\ Formation of an acyl-CoA thioester has been proposed, but not directly \\ demonstrated, to be a key step in mediating both lactonization and atypical $\beta$-oxidation of \\ 3-hydroxy-3-methylglutaryl-CoA reductase inhibitors. Here, we describe studies to \\ characterize formation of acyl-CoA thioesters in vitro in mouse liver preparations, using \\ the hydroxy acid form of simvastatin (SVA) as a model substrate. With an optimized \\ chromatography method, three new products were detected in addition to the dehydration \\ product (P1) and the lactone form of simvastatin, which have been characterized \\ previously (Prueksaritanont et al., 2001). Based on HPLC analysis, UV spectroscopy, \\ mass spectrometry and NMR spectral characterization, two metabolites were identified as \\ acyl-CoA thioester conjugates of SVA and P1, respectively, whereas the third metabolite \\ (M1) was confirmed to be the L- $\beta$-hydroxy isomer of simvastatin. M1 was likely formed \\ by stereospecific hydration, a previously reported reaction, and subsequent lactonization \\ of P1-S-acyl CoA. Among all the mouse liver subcellular fractions, microsomes exhibited \\ the highest capacity to catalyze the CoASH-dependent metabolism of SVA, while such \\ activity was totally absent in cytosol. Together, these results provide direct experimental \\ evidence that SVA (and conceivably other statins as well) is able to form an acyl-CoA \\ thioester, possibly by microsomal long-chain acyl-CoA synthetase(s), leading to \\ formation of two parallel metabolic pathways; one resulting in the two diastereomers of \\ statin lactones (simvastatin and M1), and the other to the $\beta$-oxidation pathway of statin \\ hydroxy acids.
}




\section{DMD \# 6650}

\section{Introduction}

\section{3-Hydroxy-3-methylglutaryl-coenzyme A (HMG-CoA) reductase inhibitors} (statins) are a well-established class of drugs used widely in the treatment of hypercholesterolemia due to their tolerability, proven efficacy and convenient administration. Except for simvastatin and lovastatin, all current statins are administered as the pharmacologically active hydroxy acid form. Simvastatin and lovastatin are administered as inactive lactones, which, upon hydrolysis to their respective hydroxy acids (SVA and LVA), serve as potent competitive inhibitors of HMG-CoA reductase, the rate-limiting enzyme in de novo cholesterol biosynthesis (Duggan and Vickers, 1990).

All statins, with the exception of pravastatin (Hatananka, 2000) and recently approved rosuvastatin (Martin et al., 2000), are extensively metabolized before elimination (Reinoso et al., 2002). The common metabolic pathways of these statins include P450-mediated oxidative metabolism (Igel et al., 2001), acyl glucuronidation (Prueksaritanont et al., 2002a, 2002b) and $\beta$-oxidation (Reinoso et al., 2002). Like the acyl glucuronidation, the $\beta$-oxidation occurs at the dihydroxy heptanoic or heptenoic acid side chain, a structural feature common to all statin hydroxy acids (SVA structure as an example shown in Figure 1). The pentanoic and/or propanoic acid products corresponding to one and/or two cycles of $\beta$-oxidation, respectively, have been reported to occur exclusively in rodents following lovastatin, simvastatin and cerivastatin administration and predominantly so for other statins (Vickers et al., 1990; Halpin et al., 


\section{DMD \# 6650}

1993; Boberg et al., 1998; Reinoso et al., 2002). Acyl-CoA thioesters of these statin hydroxy acids have been proposed to be the key intermediates for the formation of these $\beta$-oxidation metabolites (Figure 1). Evidence that supports this proposal, however, is very limited. Recent studies in our laboratory have provided some evidence that formation of the $\beta$-oxidation products of SVA in vitro in mouse liver preparations was mediated by CoASH-dependent enzyme(s) (Prueksaritanont et al., 2001). Unfortunately, acyl-CoA thioesters of SVA and its $\beta$-oxidation intermediates were not detected in the study. Therefore, the present studies were undertaken, using an in vitro approach and SVA as a model substrate, to characterize the formation of these acyl-CoA thioester intermediates in mouse liver subcellular fractions, and to provide support for the mechanism of the $\beta$ oxidation of statins proposed by Halpin et al. (1993). Here, we demonstrate that acylCoA conjugates of SVA and its dehydration product (P1) were formed in vitro in mouse liver preparations and were primarily catalyzed by microsomal enzymes. Additionally, the findings of CoASH-dependent formation of simvastatin and its diastereomer M1 substantiate the mechanism proposed previously for the in vivo lactonization of statin hydroxy acids (Duggan and Vickers, 1990). 


\section{DMD \# 6650}

\section{Methods}

Materials. Simvastatin and SVA were synthesized at Merck Research Laboratories (Rahway, NJ). Triton X-100, CoASH, ATP, and palmitoyl-S-acyl coenzyme A (PA-CoA) were obtained from Sigma (St. Louis, MO). $\left[1-{ }^{14} \mathrm{C}\right]-\mathrm{Palmitic}$ acid and $[1-$ $\left.{ }^{14} \mathrm{C}\right]$-octanoic acid were obtained from Amersham Bioscience (Piscataway, NJ) and American Radiolabeled Chemicals, Inc. (St. Louis, MO), respectively. All other agents were of analytical or HPLC grade.

Animals. All animal studies were approved by the Merck Research Laboratories Institutional Animal Care and Use Committee. Ten male CD-1 mice (25-40 g) were obtained from Charles River Laboratories (Wilmington, MA). Following cervical dislocation, livers were quickly removed, weighed, and washed with $1.15 \%$ potassium chloride. The livers were homogenized in 4 volumes of ice cold medium containing 250 $\mathrm{mM}$ sucrose, $10 \mathrm{mM}$ HEPES and $1 \mathrm{mM}$ EDTA. The liver subcellular fractions were prepared by differential centrifugation as described previously with minor modifications (Bjorge and Baillie, 1991). Briefly, liver homogenates were centrifuged first at $600 \mathrm{~g}$ for 10 min to remove nuclei and cell debris. The supernatant (S0.6) was centrifuged at 3,300 $g$ for a further 10 min to sediment the heavy mitochondria (MT-H). The resulting supernatant (S3) was then centrifuged at 27,000 $g$ for 10 min to obtain the light mitochondria fraction (MT-L). The corresponding supernatant (S27) was finally centrifuged at 100, $000 \mathrm{~g}$ for $1 \mathrm{hr}$ to prepare the liver microsomes. The residue supernatant was taken as the cytosolic fraction. The purity of each of the fractions was 


\section{DMD \# 6650}

assessed by the activity of their respective marker enzymes: catalase for peroxisomes (Baudhuin et al., 1964), cytochrome c oxidase for mitochondria (Sellinger et al., 1960), lactate dehydrogenase for cytosol (Volkl and Fahimi, 1985) and NADPH-cytochrome c reductase for microsomes (Beaufay et al., 1974).

In Vitro Metabolism. Incubations of SVA $(100 \mu \mathrm{M})$ with mouse liver subcellular fractions $(1.5 \mathrm{mg} / \mathrm{mL})$ were carried out in triplicate in $150 \mathrm{mM}$ Tris- $\mathrm{HCl}$ buffer (pH 8.0) containing $0.05 \%$ Triton X-100, $1.2 \mathrm{mM} \mathrm{CoASH,} 10 \mathrm{mM} \mathrm{MgCl}_{2}$, and 5 $\mathrm{mM}$ ATP in a final volume of $0.2 \mathrm{~mL}$. After a 3-min preincubation, reactions were initiated by addition of ATP and incubated for up to $60 \mathrm{~min}$ at $37^{\circ} \mathrm{C}$. The reactions were terminated at appropriate time intervals by addition of $120 \mu \mathrm{L}$ of ice cold acetonitrile. After centrifugation at $10,000 \mathrm{~g}$ for $10 \mathrm{~min}$, the supernatant was analyzed immediately by HPLC or LC-MS with an autosampler set at $5^{\circ} \mathrm{C}$ to minimize any degradation that might have occurred during the sample handling and analysis. Preliminary stability studies showed that SVA-S-acyl coenzyme A (SVA-CoA) and its subsequent metabolites were stable under the condition described above. Control experiments included incubation mixtures with one or more components missing.

Incubations with $\left[1-{ }^{14} \mathrm{C}\right]$ - palmitic acid $(50 \mu \mathrm{M})$ and $\left[1-{ }^{14} \mathrm{C}\right]-$ octanoic acid $(50$ $\mu \mathrm{M})$ were performed under the same conditions as described above for SVA, except that the mixtures contained $0.025 \mathrm{mg} / \mathrm{mL}$ of mouse liver subcellular fractions for palmitic acid and $0.125 \mathrm{mg} / \mathrm{mL}$ for octanoic acid and were incubated for $5 \mathrm{~min}$. Under such incubation conditions, the formation of PA-CoA and octanoyl-S-acyl coenzyme A (OACoA) was linear with incubation time and protein concentration. 


\section{DMD \# 6650}

For isolation and purification of metabolites of SVA, large scale incubations of SVA $(500 \mu \mathrm{M})$ were carried out with mouse liver microsomes $(1.5 \mathrm{mg} / \mathrm{mL})$, CoASH $(1.2$ $\mathrm{mM}$ ) and ATP (5 mM) for $40 \mathrm{~min}$ in a final volume of $12 \mathrm{~mL}$ for the isolation of SVACoA and P1-S-acyl coenzyme A (P1-CoA) or for $3 \mathrm{hr}$ in a final volume of $60 \mathrm{~mL}$ for M1. The incubation mixtures were terminated with half a volume of acetonitrile and loaded onto $\mathrm{C}_{18}$ solid phase extraction cartridges (Oasis HLB SPE cartridge, Waters Corporation, Milford, MA), which were pre-equilibrated with water and acetonitrile. The acyl-CoA thioesters-containing fractions were eluted with $70 \%$ acetonitrile, while M1containing fraction was eluted with $90 \%$ acetonitrile. The resulting solid phase eluates were evaporated to dryness, reconstituted in $5-10 \mathrm{~mL}$ of the mobile phase $(20 \%$ acetonitrile in $10 \mathrm{mM}$ ammonium acetate buffer, $\mathrm{pH}=7$ ) and isolated by preparative HPLC as described below. The purified metabolites were characterized by NMR spectroscopy.

Analytical Methods. SVA and its metabolites were analyzed using published HPLC methods with some modifications (Prueksaritanont et al., 1999). In brief, samples held in an autosampler (set at $5^{\circ} \mathrm{C}$ ) were chromatographed on a $\mathrm{C}_{18}$ Zorbax column (150 x $4.6 \mathrm{~mm}, 5 \mu \mathrm{m}$; Agilent Technologies, Foster City, CA) with a linear gradient of acetonitrile and $10 \mathrm{mM}$ ammonium acetate $(\mathrm{pH} 7)$. The eluates were monitored by UV absorption at $240 \mathrm{~nm}$ and $260 \mathrm{~nm}$. Due to the unavailability of authentic standards for SVA-CoA and P1-CoA, the concentrations of these metabolites in the in vitro incubation mixtures were estimated using standard curves for PA-CoA at $260 \mathrm{~nm}$, assuming similar extinction coefficients between SVA-CoA (or P1-CoA) and PA-CoA. Evidence that 


\section{DMD \# 6650}

supports the assumption includes the observations that SVA, P1 and palmitic acid all had minimal UV absorption at $260 \mathrm{~nm}$, whereas the corresponding acyl-CoA thioesters and free CoASH exhibited maximal UV absorption at $260 \mathrm{~nm}$. In addition, PA-CoA was selected because of its similar HPLC retention time to SVA-CoA and P1-CoA under the HPLC conditions described above. Quantification of P1 and M1 in the in vitro incubations was accomplished using standard curves for SVA and simvastatin, respectively, assuming identical extraction recoveries and extinction coefficients at their maximal UV absorption at $240 \mathrm{~nm}$.

Metabolite isolation was accomplished by semi-preparative HPLC (Waters, Milford, MA) coupled with a fraction collector (Foxy 200; ISCO, Inc., Lincoln, NE). The reconstituted reaction mixtures were separated on a Luna $\mathrm{C}_{18}(2)$ column $(250 \mathrm{x} 10 \mathrm{~mm}, 5$ $\mu \mathrm{m}$; Phenomenex, Torrance, CA) with a 40-min gradient from $80 \%$ A (10\% methanol in $10 \mathrm{mM}$ ammonium acetate, $\mathrm{pH} 7)$ to $70 \% \mathrm{~B}$ (90\% acetonitrile in water). Fractions corresponding to the peaks for the SVA metabolites were collected according to UV absorbance at $260 \mathrm{~nm}$ for SVA-CoA and P1-CoA and $240 \mathrm{~nm}$ for M1.

Analysis of PA-CoA and OA-CoA in mouse liver preparations was performed on a BDS Hypersil $C_{8}$ coulmn $(150$ x 4.6 mm, $5 \mu \mathrm{m}$; Thermo Hypersil-Keystone, Bellefonte, PA) with a linear gradient of acetonitrile and $25 \mathrm{mM}$ ammonium acetate (pH 7). The acyl-CoA thioesters were monitored by UV absorption at $260 \mathrm{~nm}$ and by an on-line IN/US $\beta$-ram radioactivity detector (IN/US Systems, Tampa, FL).

LC-MS analysis was conducted on a Finnigan MAT LCQ ion trap mass spectrometer (Thermo Finnigan, San Jose, CA), interfaced with a PerkinElmer HPLC system (PerkinElmer Life and Analytical Sciences, Boston, MA) equipped with two 


\section{DMD \# 6650}

Series 200 micro pumps and an autosampler set at $5^{\circ} \mathrm{C}$. LC-MS and LC-MS/MS

experiments were carried out using electrospray ionization in the negative ion mode (for SVA, P1, SVA-CoA, and P1-CoA ) or positive ion mode (for lactones: simvastatin and M1). The electrospray ionization voltage was set at $3.5 \mathrm{kV}$ with the heated capillary temperature held at $175^{\circ} \mathrm{C} . \mathrm{A} \mathrm{C}_{18}$ Zorbax column $(150$ x $4.6 \mathrm{~mm}, 5 \mu \mathrm{m}$; Agilent Technologies, Foster City, CA) was used for chromatographic separation and was eluted at $1.0 \mathrm{~mL} / \mathrm{min}$ with a gradient of $10 \mathrm{mM}$ ammonium acetate $(\mathrm{pH} 7)$ in water with $10 \%$ methanol (A) and 90\% acetonitrile in water (B). The initial mobile phase consisted of $15 \% \mathrm{~B}$, which remained unchanged for $5 \mathrm{~min}$, and increased linearly to $70 \% \mathrm{~B}$ over 22 min, then to $90 \%$ over $1 \mathrm{~min}$, and held for $5 \mathrm{~min}$. One fifth of the flow was directed into the mass spectrometer, and the remainder was used for UV detection at $240 \mathrm{~nm}$.

Accurate mass measurements were conducted on a Micromass Q-TOF (quadrupole time-of-flight) II mass spectrometer (Waters, Milford, MA) operated in the positive electrospray ionization mode with a LockSpray reference sprayer. The source and desolvation gas temperatures were set at $80^{\circ} \mathrm{C}$ and $180^{\circ} \mathrm{C}$, respectively. The capillary voltage, cone voltage and collision energies were $2.5 \mathrm{kV}, 25 \mathrm{~V}$ and $15 \mathrm{~V}$, respectively. The instrument was calibrated by infusing aqueous $0.01 \%$ phosphoric acid solution through a syringe pump at $5 \mu \mathrm{L} / \mathrm{min}$ prior to the experiment. The same calibration solution was used as lock mass in the sample analysis. The chromatographic conditions used in Q-TOF experiments were the same as described in LCQ section.

NMR studies were performed at $10^{\circ} \mathrm{C}$ for $\mathrm{CoASH}$ and acyl-CoA thioesters, and at $25{ }^{\circ} \mathrm{C}$ for the SVA, simvastatin, and M1 samples. All NMR data were collected either on a 500 or $600 \mathrm{MHz}$ spectrometer (Varian Inc., Palo Alto, CA) both equipped with a $5 \mathrm{~mm}$ 


\section{DMD \# 6650}

HCN Cold Probe (Varian Inc.). The HPLC isolates or the authentic standard(s) were dissolved in $\sim 160 \mu \mathrm{L}$ of $\mathrm{CD}_{3} \mathrm{OD}$ (except for $\mathrm{CoASH}$, where $10 \% \mathrm{D}_{2} \mathrm{O}$ v/v was added to promote solubility) and transferred to $3 \mathrm{~mm}$ tubes. 1D ${ }^{1} \mathrm{H}, 2 \mathrm{D}{ }^{1} \mathrm{H}-{ }^{1} \mathrm{H}$ correlation, 2D ${ }^{1} \mathrm{H}$ -

${ }^{13} \mathrm{C}$ one-bond and multiple-bond correlation data sets were acquired for all samples and used to elucidate the structures. 


\section{DMD \# 6650}

\section{Results}

Metabolite Identification. Figures 2A and 2B illustrate typical HPLC chromatograms derived from incubations of mouse liver subcellular fractions (S0.6 and microsomes) with SVA in the presence of CoASH and ATP. Five metabolites (designated as SVA-CoA, P1-CoA, P1, M1, and simvastatin) were detected by UV absorption at $240 \mathrm{~nm}$. P1, M1 and simvastatin exhibited UV absorption spectra similar to SVA, whereas SVA-CoA and P1-CoA had UV absorption peak at $260 \mathrm{~nm}$ (characteristics for CoASH) in addition to the well-characterized UV peak for SVA at $240 \mathrm{~nm}$. P1, which gave a deprotonated ion $[\mathrm{M}-\mathrm{H}]^{-}$at $m / z, 417$, has been identified previously by NMR as the dehydration product of SVA (Prueksaritanont et al., 2001). The product, which eluted last in the chromatogram, was identified as the simvastatin lactone based on the identical HPLC retention time and UV spectrum compared with the authentic standard. Except for a small amount of simvastatin $(<0.1 \%$ of initial concentration), the remaining four SVA metabolites were not detectable in incubations with mouse liver cytosol (Figure 2C) and control incubations that lacked liver protein, ATP, or CoASH. The results suggested that formation of SVA-CoA, P1-CoA, P1, and M1 was mediated by CoASH- and ATP-dependent enzyme(s), whereas simvastatin was formed by both enzymatic (by CoASH- and ATP-dependent enzymes) and chemical (to much less extent) processes under the conditions decribed in the methods.

The mass spectrum of SVA-CoA showed a prominent $[\mathrm{M}-\mathrm{H}]^{-}$ion at $\mathrm{m} / \mathrm{z}$ 1184.4, $749\left(\mathrm{CoASH}-\mathrm{H}_{2} \mathrm{O}\right)$ mass units higher than the $[\mathrm{M}-\mathrm{H}]^{-}$ion of SVA. MS/MS fragmentation of $m / z 1184.4$ showed peaks indicative of a CoASH moiety attached to SVA, including ions at $\mathrm{m} / \mathrm{z} 766.2$ ([CoAS] $\left.{ }^{-}\right), \mathrm{m} / \mathrm{z} 426.1$ ([adenosine diphosphate] $\left.]^{-}\right), \mathrm{m} / \mathrm{z}$ 


\section{DMD \# 6650}

408.2 ([adenosine diphosphate $-\mathrm{H}_{2} \mathrm{O}^{-}$),$m / z 1104.5$ ([SVA-CoA $\left.-\mathrm{H}_{2} \mathrm{PO}_{3}\right]^{-}$), $m / z, 1086.5$ ([SVA-CoA - dimethyl-oxobutyl] $\left.]^{-}\right), m / z$ 1049.4 ([SVA-CoA - adenine - 2H] $]^{-}$), and $m / z$ 837.4 ([SVA-CoA - adenosine monophosphate - 2H]') (Figure 3A). NMR studies with the isolated metabolite confirmed it to be an acyl-CoA conjugate of SVA with a thioester bond between SVA and CoASH. The combined NMR data clearly showed that the SVA and CoASH moieties were fully intact in the adduct spectrum. The methylene group, labeled 3 , in the SVA portion of the adduct appeared at $\delta_{\mathrm{H}} 2.71,2.77 \mathrm{ppm} / \delta_{\mathrm{C}} 50.8 \mathrm{ppm}$ (Table 1). The $\beta$-methylene group, labeled a', of the mercaptoethylamine subunit in CoASH appeared at $\delta_{\mathrm{H}} 3.00 \mathrm{ppm} / \delta_{\mathrm{C}} 27.9 \mathrm{ppm}$ (Table 1). A $2 \mathrm{D}{ }^{1} \mathrm{H}_{-}{ }^{13} \mathrm{C}$ multi-bond correlation (a select expansion of the spectrum displayed in Figure 4) was observed from both methylene protons $\mathbf{3}$ and $\mathbf{a}$ ' to the carbonyl carbon $\mathbf{2}$ (at $197.9 \mathrm{ppm}$ ) in the SVA heptanoic acid side chain. Therefore, the combined NMR data showed that the sulfur of the CoASH was conjugated to the acyl carbon $\mathbf{2}$ of the heptanoic acid side chain in SVA.

The identification of P1-CoA was based on UV, MS, MS/MS, and NMR spectra. P1-CoA gave rise to a deprotonated ion $[\mathrm{M}-\mathrm{H}]^{-}$at $m / z$ 1166.4, $749\left(\mathrm{CoASH}-\mathrm{H}_{2} \mathrm{O}\right)$ mass units higher than the $[\mathrm{M}-\mathrm{H}]^{-}$ion of $\mathrm{P} 1$ and 18 mass units less than that of SVACoA thioester $(m / z$ 1184.4), suggesting possible dehydration of SVA-CoA. Consistently, the MS/MS spectrum of P1-CoA displayed a family of product ions, including ions $\mathrm{m} / \mathrm{z}$ 1086.5 ([P1-CoA - $\left.\left.\mathrm{H}_{2} \mathrm{PO}_{3}\right]^{-}\right), m / z 1068.5$ ([P1-CoA - dimethyl-oxobutyl] $\left.{ }^{`}\right), m / z 1031.4$ ([P1-CoA - adenine - 2H $\left.]^{-}\right)$, and $m / z 819.4$ ([P1-CoA - adenosine monophosphate $2 \mathrm{H}^{-}$) (Figure 3B), which were all 18 mass units less than the corresponding fragment ions of SVA-CoA. In addition, the product ions at $m / z 426.1$ and 408.2, corresponding to adenosine diphosphate and its dehydration ions, remained the same as those of SVA- 


\section{DMD \# 6650}

CoA, indicating that the adenosine phosphate moiety was intact (Figure 3 B). NMR data supported that P1-CoA was an acyl-CoA conjugate of P1 with a thioester bond between $\mathrm{P} 1$ and CoASH. The combined NMR data conclusively indicated that the P1 and CoASH moieties were intact. The proton shifts of P1 moiety were similar to the previously published data on P1, the dehydration metabolite of SVA (Prueksaritanont et al., 2001). Alkene carbons 3 and 4 in the P1 moiety appeared at $\delta_{\mathrm{H}} 6.22 \mathrm{ppm} / \delta_{\mathrm{C}} 129.9 \mathrm{ppm}$ and $\delta_{\mathrm{H}}$ $6.90 \mathrm{ppm} / \delta_{\mathrm{C}} 142.6 \mathrm{ppm}$, respectively (Table 1). The $\beta$-methylene group, labeled $\mathbf{a}$, of the mercaptoethylamine subunit in CoASH appeared at $\delta_{\mathrm{H}} 3.04 \mathrm{ppm} / \delta_{\mathrm{C}} 27.6 \mathrm{ppm}$ (Table 1). A common $2 \mathrm{D}^{1} \mathrm{H}_{-}{ }^{13} \mathrm{C}$ multi-bond correlation was observed from the protons 3 and $\mathbf{4}$ of P1 moiety and methylene protons $\mathbf{a}$ ' to the carbonyl carbon $\mathbf{2}$ at $190.0 \mathrm{ppm}$ (not shown). Therefore, NMR data conclusively indicated the site of CoASH sulfur conjugation to be at the acyl carbon 2 of P1 moiety.

M1 was identified as L- $\beta$-hydroxy isomer of simvastatin based on UV, accurate MS, MS/MS, and NMR spectra. Accurate mass measurements by a Q-TOF instrument revealed that the $[\mathrm{M}+\mathrm{H}]^{+}$of simvastatin standard and M1 were at $m / z 419.2788$ and 419.2804, respectively, which were all within $2.2 \mathrm{ppm}$ of the calculated mass of $[\text { simvastatin }+\mathrm{H}]^{+}$. In the high resolution MS/MS experiment the residual parent ion signal was used as the internal lock mass. MS/MS spectra of simvastatin and M1 exhibited almost identical fragmentation patterns except for the relative intensities of fragment ions (not shown). The observed and calculated $\mathrm{m} / \mathrm{z}$ values of the most abundant fragment ions of simvastatin and M1 are shown in Table 2, which confirmed the fragmentation pathway proposed in Figure 5. Therefore, high resolution MS data strongly suggested that simvastatin and M1 were positional isomers. The NMR data on M1 was 


\section{DMD \# 6650}

consistent with a chiral center inversion at carbon $\mathbf{4}$ that is part of the six-membered lactone ring. ${ }^{1} \mathrm{H}$ chemical shifts of simvastatin standard and $\mathrm{M} 1$ are shown in Table 1. Comparing the two 1D ${ }^{1} \mathrm{H}$ NMR spectra, the spectral changes were observed for protons in the six-membered lactone ring. The $\mathrm{CHOH}$ methine protons, labeled $\mathbf{4}$ and $\mathbf{6}$, appeared at 4.24 and $4.61 \mathrm{ppm}$, respectively, in simvastatin (Table 1). In contrast, H4 and H6 were overlapped and appeared at $4.19 \mathrm{ppm}$ in the M1 spectrum (Table 1). The methine carbons at 4 and 6 were resolved in the $2 \mathrm{D}{ }^{1} \mathrm{H}^{-13} \mathrm{C}$ one-bond correlation spectrum of M1 at 62.8 ppm and $77.8 \mathrm{ppm}$, respectively (not shown).

Metabolism of SVA in mouse liver subcellular fractions. Time-dependent studies with mouse liver S0.6 showed that formation of SVA-CoA and P1-CoA increased rapidly during the first 10-20 min incubations and then decreased steadily thereafter, whereas formation of P1, M1, and simvastatin appeared to increase gradually with incubation time (Figure 6A). In addition to mouse liver S0.6, CoASH-dependent metabolism of SVA was also observed in incubations with other mouse liver subcellular fractions, including mitochondria (MT-H and MT-L) and microsomes (Figure 7C). The identities and purities of these subcellular fractions were confirmed by marker enzyme analysis (Table 3). Among all the mouse subcellular fractions, microsomes exhibited the highest capability to catalyze CoASH-dependent metabolism of SVA on a per mg protein basis, whereas cytosol was totally incapable of catalyzing the reaction (Figures $2 \mathrm{C}$ and 7C). As illustrated in Figures $2 \mathrm{~B}$ and 6B, incubations of SVA with mouse liver microsomes produced significantly higher levels of SVA-CoA and P1-CoA thioesters (approximately 5 to 7 fold higher after 20 min incubations) than that of mouse liver S0.6. Consistently, the lactonization products, simvastatin and M1, were also found to be 


\section{DMD \# 6650}

approximately 5 to 7 fold higher after 1-hr incubations with mouse liver microsomes than the corresponding incubations with S0.6. These results provided additional support for the acyl-CoA thioester-dependent mechanism proposed previously for the lactonization of the hydroxy acid forms of statins observed in vivo (Duggan and Vickers, 1990). Interestingly, P1 levels in microsomes were not significantly more than in S0.6.

Due to the apparent subsequent metabolism of SVA-CoA in mouse liver preparations, the sum of the five metabolites (SVA-CoA, P1-CoA, P1, M1, and simvastatin) was used to compare the rates of CoASH-dependent metabolism of SVA in mouse liver subcellular fractions. Preliminary studies showed that CoASH-dependent metabolism of SVA was linear with time up to $30 \mathrm{~min}$ and with mouse liver S0.6 up to 3 $\mathrm{mg} / \mathrm{mL}$. As shown in Figure 7C and Table 3, the rate of CoASH-dependent metabolism of SVA in mouse liver subcellular fractions appeared to correlate well with that of acylCoA formation of palmitic acid (a substrate for long-chain acyl-CoA synthetase) (Figure 7 A) and the activity of cytochrome c reductase, a marker enzyme for microsomes, but not with the rate of acyl-CoA formation of octanoic acid (a substrate for medium-chain acyl-CoA synthetase) (Figure 7B) and the activities of marker enzymes for other subcellular fractions. These observations clearly indicated that acyl-CoA formation of SVA is catalyzed preferentially by microsomal enzyme(s). 


\section{DMD \# 6650}

\section{Discussion}

The present studies provide direct experimental evidence for the formation of acyl-CoA thioester of SVA in vitro in mouse liver preparations. Employing the HPLC methods described above, acyl-CoA thioester intermediates of SVA were completely resolved from other peaks present following incubations with mouse liver preparations. Under such HPLC conditions optimized for acyl-CoA thioesters, we were able to isolate and fully characterize these acyl-CoA thioester intermediates by LC-MS/MS and NMR analysis. The optimized HPLC method also provides a possible explanation for the reason that the CoA thioester conjugates of SVA were not observed in our previous studies with mouse liver preparations (Prueksaritanont et al., 2001). Preliminary studies showed that SVA-CoA and P1-CoA were sensitive to $\mathrm{pH}$ of mobile phase and did not chromatograph well under acidic conditions, such as $0.1 \%$ formic acid, the conditions used in our previous studies (Prueksaritanont et al., 2001). Similar observations were also made for other xenobiotic acyl-CoA thioesters, such as 2-phenylpropionyl-S-acyl CoA (Li et al., 2002), 2,4-dichlorophenoxyacetyl-S-acyl CoA (Li et al., 2003) and naproxen-Sacyl CoA (Olsen et al., 2002). Recently, Sidenius el al. (2004) reported several LC chromatographic methods developed for analysis of acyl-CoA thioesters of eight structurally diverse carboxylic acids, including ibuprofen, clofibric acid, indomethacin, fenbufen, tolmetin, salicylic acid, 2-phenoxypropionic and 4-chloro-2-methylphenoxyacetic acid. Notably, the $\mathrm{pH}$ of mobile phases used for the eight acyl-CoA thioesters were all between 6 and 7. 


\section{DMD \# 6650}

As pointed out by Halpin et al. (1993), $\beta$-oxidation of fatty acids requires that hydroxylated intermediates be in the L-configuration, while in the case of statins, both the 4- and 6-hydroxyl groups on the side chain possess the D-configuration required for pharmacological activity (Figure 1). An epimerization to L-configuration has been proposed to be an essential step for D- $\beta$-hydroxyacyl CoA thioester to enter the $\beta$ oxidation cycles (Halpin et al., 1993) (Figure 1). Recent in vitro studies with SVA provided evidence supporting that epimerization of D- $\beta$-hydroxy statin acyl CoA proceeded via the dehydration at the $\alpha$ and $\beta$ carbons with respect to the carboxy carbon to give a trans-enoyl-CoA, followed by stereospecific hydration of the unsaturated acylCoA to give rise to the L- $\beta$-hydroxy isomer (Prueksaritanont et al., 2001) (Figure 1). However, the corresponding dehydration (P1) and stereospecific rehydration (P2: L,Ddihydroxy isomer of SVA) intermediates were detected only in their free acid forms (Prueksaritanont et al., 2001). Under the current HPLC conditions, we were able to detect the acyl-CoA thioester of the dehydration product, namely P1-CoA, in addition to its free acid form P1. However, the peak corresponding to acyl-CoA thioester of P2 (P2-S-acyl coenzyme A, P2-CoA) was not observed in the present study. The reason for this remains unclear. It is possible that formation of P2-CoA is low under current incubation conditions and could not be detected by HPLC analysis.

In addition to mediating the $\beta$-oxidation of statins, the bioactivated acyl-CoA thioesters have been hypothesized to be a key intermediate for the formation of statin lactones (Duggan and Vickers, 1990). The hypothesis has been substantiated by recent findings of $\mathrm{CoASH}$-dependent formation of simvastatin lactone in vitro in mouse liver preparations (Prueksaritanont et al., 2001). Similar observations on simvastatin formation 


\section{DMD \# 6650}

were also made in the present study. In addition to simvastatin, we found a new lactone metabolite of SVA, M1 (L- $\beta$-hydroxy isomer of simvastatin), possibly formed by lactonization of P2-CoA, and its structure has been identified definitively (Figure 1). Our finding that the extent of formation of simvastatin and M1 increased when the corresponding acyl-CoA formation (SVA-CoA and P1-CoA) increased (Figure 6) provides additional evidence that the lactonization of the statin hydroxy acids (SVA and P2) could be mediated by acyl-CoA thioesters via an intramolecular acylation.

Considering the well-documented interconversion between SVA and simvastatin, it is anticipated that interconversion between P2 and M1 might also exist (Figure 1). Both P2 and M1 are pharmacologically inactive, and the relevance of the in vitro formation of M1 to in vivo situation is currently unclear. The formation of M1 has not been reported in vivo following administration of SVA or simvastatin to humans and animals. This might be partly due to the chromatographic conditions used in the previous in vivo studies, which were not optimized for the detection of M1. It is also possible that formation of $\mathrm{M} 1$ is relatively low in vivo if the subsequent $\beta$-oxidation of $\mathrm{P} 2-\mathrm{CoA}$ is more favorable than the corresponding lactonization process (Figure 1).

In agreement with previous data (Krisans et al., 1980 and Knights and Drogemuller, 2000), our study showed that long chain acyl-CoA synthetase (measured by PA-CoA synthetase) was primarily localized in microsomes and to less extent in mitochondria (MT-H and MT-L) (Figure 7A), while medium chain acyl-CoA synthetase (measured by OA-CoA synthetase) was mainly located in mitochondria (MT-H and MTL) (Figure 7B), which further confirmed the purity of subcellular fractions obtained. Studies with these subcellular fractions revealed that CoASH-dependent SVA 


\section{DMD \# 6650}

metabolism occurred preferentially in microsomes and to much less extent in mitochondria (MT-H and MT-L) with no activity observed in cytosol (Figure 7C). This distribution of activity correlated well with that of long chain acyl-CoA synthetase, but not with that of medium chain acyl-CoA synthetase, suggesting the potential involvement of long chain acyl-CoA synthetase in the acyl-CoA formation of SVA. This observation was further supported by our preliminary studies which showed that CoASH-dependent metabolism of SVA was effectively inhibited by palmitic acid and triacsin C (10 $\mu \mathrm{M})$, a potent and selective inhibitor for long-chain acyl-CoA synthetase (Vessey et al., 2004). In contrast, the enzymes potentially involved in the hydrolysis of SVA-CoA and P1-CoA were still poorly understood. It appeared that other subcellular fractions (e.g. mitochondria and cytosol), in addition to microsomes, might contribute significantly to the hydrolysis of P1-CoA, based on the fact that P1 levels were not significantly increased when the corresponding P1-CoA was increased by 5-7 fold in liver microsomes as compared to liver S0.6 (Figure 6). This observation is consistent with the previous reports on the wide subcelluar distribution of acyl-CoA hydrolases (Urrea and Bronfman, 1996 and Garras et al., 1997).

It is noteworthy that formation of statin acyl-CoA thioesters is not expected to be unique for SVA. Other statin hydroxy acids, including cerivastatin and atorvastatin, which all share the common D,D-dihydroxy acid side chain undergo $\beta$-oxidation, and therefore, are anticipated to be able to form the acyl-CoA thioesters. In fact, our preliminary studies with cerivastatin and atorvastatin showed that acyl-CoA thioesters of these statin acids were readily detectable in vitro in mouse liver preparations. Additionally, consistent with the in vivo observations on the $\beta$-oxidation of statins 


\section{DMD \# 6650}

(Reinoso et al., 2002), species differences in the formation of acyl-CoA thioesters were also observed in our preliminary studies, which showed that acyl-CoA thioesters of statin hydroxy acids, including SVA, cerivastatin and atorvastatin, were detectable only with liver preparations from mice and rats, but not from dogs and humans. However, such marked species differences were not observed in vivo for the formation of statin lactones. Significant lactone formation was reported to occur in vivo following administration of SVA or cerivastatin to dogs and of cerivastatin and atorvastatin to humans (Vickers et al., 1990; Boberg et al., 1998; Kantola et al., 1998, 1999), in addition to rodents, suggesting that more than one metabolic pathway might contribute to the formation of statin lactones. In fact, recent study showed that acyl glucuronides also mediated the lactone formation (Prueksaritanont et al., 2002a). Quantitatively, there appears to be differences in the relative contribution of these two metabolic pathways to the lactone formation of different statins in different species. In rodents, acyl-CoA pathway is likely to contribute significantly to the formation of statin lactones based on the fact that $\beta$-oxidation of statins has been shown to be a major pathway in these species (Reinoso et al., 2002). Conversely, in dogs, acyl glucuronidation appears to play a more important role in the lactonization of statins than acyl-CoA formation; statins have been shown to undergo significant acyl glucuronidation and minimal $\beta$-oxidation in vivo in dogs (Vickers et al., 1990; Boberg et al., 1998; Black et al., 1999; Prueksaritanont et al., 2002a). Unfortunately, such conclusion could not be readily drawn in humans, due to limited in vivo data. Nevertheless, based on the findings on acyl glucuronidation in human liver preparations in vitro and in animals in vitro and in vivo (Prueksaritanont et al., 2002a), it 


\section{DMD \# 6650}

is anticipated that acyl glucuronidation plays a role in the lactonization of statin acids in vivo in humans.

In conclusion, this was the first study to demonstrate the formation of acyl-CoA thioesters of SVA and subsequent metabolites in vitro in mouse liver preparations. Our results support the hypothesis that $\beta$-oxidation occurs as a consequence of acyl-CoA thioester formation, possibly by microsomal long-chain acyl-CoA synthetase(s), and subsequent epimerization. Additionally, the present studies also provide evidence that formation of these thioesters contributes, at least in part, to interconversion between SVA and its diastereomer and between these hydroxy acids and their corresponding lactones. Similar observations are also anticipated with other hydroxy acid forms of statins (and their diastereomers) and the corresponding lactones.

\section{Acknowledgements}

We thank Scott Fauty and Todd Killino for mouse liver preparations and Dr. Don Slaughter for critical review of the manuscript. 


\section{DMD \# 6650}

\section{References}

Aas M (1971) Organ and subcellular distribution of fatty acid activating enzymes in the rat. Biochim Biophys Acta 231: 32-47.

Baudhuin P, Beaufay H, Rahman LO, Selliner OZ, Wattiaux R, Jacques P and de Duve C (1964) Tissue fractionation studies. Biochem J 92: 179-184.

Beaufay H, Amar-Cortesec A, Feytmans E, Thines-Sempoux D, Wibo M, Mobbi MN and Berhet T (1974) Analytical study of microsomes and isolated subcellular membranes from rat liver. 1. Biochemical methods. J Cell Biol 61: 188-200.

Bjorge SM and Baillie TA (1991) Studies of the $\beta$-oxidation of valproic acid in rat liver mtiochondrial preparations. Drug Metab Dispos 19: 823-829.

Black AE, Hayes RN, Roth BD, Woo P and Woolf TF (1999) Metabolism and excretion of atorvastatin in rats and dogs. Drug Metab Dispos 27: 916-923.

Boberg M, Angebauer R, Kanhai WK, Karl W, Kern A, Radtke M and Steinke W (1998) Biotransformation of cerivastatin in mice, rats and dogs in vivo. Drug Metab Dispos 26: $640-652$.

Duggan DE and Vickers S (1990) Physiological disposition of HMG-CoA-reductase inhibitors. Drug Metab Rev 22: 333-362. 


\section{DMD \# 6650}

Garras A, Elholm M, Sleboda J, Froyland L, Osmundsen H and Berge RK (1997) On the effects of thia fatty acid analogues on hydrolases involved in the degradation of metabolisable and non-metabolisable acyl-CoA esters. Xenobiotica 27: 781-799.

Halpin RA, Ulm EH, Till AE, Kari PH, Vyas KP, Hunninghake DB and Duggan DE (1993) Biotransformation of lovastatin V. Species differences in in vivo metabolite profiles of mouse, rat, dog, and human. Drug Metab Dispos 21: 1003-1011.

Hatanaka T (2000) Clinical pharmacokinetics of pravastatin. Mechanisms of pharmacokinetics events. Clin Pharmacokinet 39: 397-412.

Igel M, Sudhop T and vonBergmann K (2001) Metabolism and drug interaction of 3hydroxy-3-methylglutaryl coenzyme A-reductase inhibitors (statins). Eur J Clin Pharmacol 57: 357-364.

Kantola T, Kivisto KT and Neuvonen PJ (1998) Effect of itraconazole on cerivastatin pharmacokinetics. Eur J Clin Pharmacol 54: 851-855.

Kantola T, Kivisto KT and Neuvonen PJ (1999) Effect of itraconazole on the pharmacokinetics of atorvastatin. Clin Pharmacokinet Ther 64: 58-65.

Knights KM and Drogemuller CJ (2000) Xenobiotic-CoA ligases: kinetic and molecular characterization. Curr Drug Metab 1: 49-66.

Krisans SK, Mortensen RM and Lazarow PB (1980) Acyl-CoA synthetase in rat liver peroxisomes. J Biol Chem 255: 9599-9607. 


\section{DMD \# 6650}

Li C, Benet LZ and Grillo MP (2002) Enantioselective covalent binding of 2-

phenylpropionic acid to protein in vitro in rat hepatocytes. Chem Res Toxicol 15: 14801487.

Li C, Grillo MP and Benet LZ (2003) In vitro studies on the chemical reactivity of 2,4dichlorophenoxyacetyl-S-acyl-CoA thioester. Toxicol Appl Pharmacol 187: 101-109.

Martin PD, Dane AL, Scheneck DW and Warwick MJ (2000) Disposition of new HMGCoA reductase inhibitor ZD4522 following dosing in healthy subjects. J Clin Pharmacol 40: 1056.

Olsen J, Bjornsdottir I, Tjornelund J and Hansen SH (2002) Chemical reactivity of the naproxen acyl glucuronide and the naproxen coenzyme A thioester towards bionucleophiles. J Pharma Biomed Anal 29: 7-15.

Prueksaritanont T, Ma B, Tang C, Meng Y, Assang C, Lu P, Reider PJ, Lin JH and Baillie TA (1999) Metabolic interactions between mibefradil and HMG-CoA reductase inhibitors: an in vitro investigation with human liver preparations. Br J Clin Pharmacol 47: 291-298.

Prueksaritanont T, Ma B, Fang X, Subramanian R, Yu J and Lin JH (2001) $\beta$-oxidation of simvastatin in mouse liver preparations. Drug Metab Dispos 29: 1251-1255.

Prueksaritanont T, Subramanian R, Fang X, Ma B, Qiu Y, Lin JH, Pearson PG and Baillie TA (2002a) Glucuronidation of statins in animals and humans: A novel mechanism of statin lactonization. Drug Metab Dispos 30: 505-512. 


\section{DMD \# 6650}

Prueksaritanont T, Tang C, Qiu Y, Mu L, Subramanian R and Lin JH (2002b) Effects of fibrates on metabolism of statins in human hepatocytes. Drug Metab Dispos 30: 12801287.

Reinoso RF, Navarro AS, Garcia MJ and Prous JR (2002) Preclinical pharmacokinetics of statins. Methods Find Exp Clin Pharmacol 24: 593-613.

Sellinger OZ, Beaufay H, Jacques P, Doyer A and de Duve C (1960) Tissue fractionation studies. Biochem J 74: 450-456.

Sidenius U, Skonberg C, Olsen J and Hansen SH (2004) In vitro reactivity of carboxylic acid-CoA thioesters with glutathione. Chem Res Toxicol 17: 75-81.

Vessey DA, Kelly M and Warren RS (2004) Characterization of triacsin C inhibition of short-, medium-, and long-chain fatty acid:CoA ligases of human liver. J Biochem Mol Toxicol 18: 100-106.

Urrea R and Bronfman M (1996) Species differences in the intracellular distribution of ciprofibryl-CoA hydrolase. Implications for peroxisome proliferation. FEBS Lett 389: 219-223.

Vickers S, Duncan CA, Chen I-W, Rosegay A and Duggan DE (1990) Metabolic disposition studies of simvastatin, a cholesterol-lowering prodrug. Drug Metab Dispos 18: $138-145$.

Volkl A and Fahimi HD (1985) Isolation and characterization of peroxisomes from the liver of normal untreated rats. Eur J Biochem 149: 257-265. 


\section{DMD \# 6650}

\section{Footnotes}

Send reprint requests to: Dr. Chunze Li, Department of Drug Metabolism, Merck

Research Laboratories, WP 75-100, P. O. Box 4, West Point, PA 19486.

${ }^{1}$ Present address: Amgen Inc., One Amgen Center Drive, Thousand Oaks, CA 91320-

1799 


\section{DMD \# 6650}

\section{Figure Legends}

Figure 1. Proposed pathway for CoASH-dependent metabolism of SVA.

Figure 2. Representative HPLC-UV profiles of SVA metabolites in incubations with mouse liver S0.6 (A), microsomes (B) and cytosol (C).

Incubations were carried out at $37^{\circ} \mathrm{C}$ for 20 min using mouse liver subcellular fractions $(1.5 \mathrm{mg} / \mathrm{mL})$ and SVA $(100 \mu \mathrm{M})$ with $1.2 \mathrm{mM} \mathrm{CoASH}$ and $5 \mathrm{mM}$ ATP.

Figure 3. MS/MS spectra of SVA-CoA (A) and P1-CoA (B) and tentative assignments of the fragment ions.

Figure 4. Select expansion of a $2 \mathrm{D}^{1} \mathrm{H}_{-}{ }^{13} \mathrm{C}$ multi-bond correlation NMR spectrum of SVA-CoA. Labels are shown in Figure 1.

Figure 5. Proposed fragmentation pathway for simvastatin and M1. The accurate mass measurement data are summarized in Table 2.

Figure 6. Time-dependent formation of SVA metabolites following incubations with mouse liver S0.6 (A) and microsomes (B).

Incubations were carried out in triplicate at $37^{\circ} \mathrm{C}$ using mouse liver subcellular fractions $(1.5 \mathrm{mg} / \mathrm{mL})$ and SVA $(100 \mu \mathrm{M})$ with $1.2 \mathrm{mM}$ CoASH and $5 \mathrm{mM}$ ATP. Values represent means \pm SD from triplicate incubations.

Figure 7. Mouse liver subcellular distribution of enzyme activity responsible for the formation of PA-CoA (A), OA-CoA (B) and CoA-dependent metabolism of SVA (C). 


\section{DMD \# 6650}

Incubations of SVA $(100 \mu \mathrm{M})$ with mouse liver subcellular fractions $(1.5 \mathrm{mg} / \mathrm{mL})$ were carried out in triplicate at $37^{\circ} \mathrm{C}$ for $20 \mathrm{~min}$ in $150 \mathrm{mM}$ Tris- $\mathrm{HCl}$ buffer $(\mathrm{pH} \mathrm{8.0)}$

containing $1.2 \mathrm{mM}$ CoASH and $5 \mathrm{mM}$ ATP. Incubations with $\left[1-{ }^{14} \mathrm{C}\right]-$ palmitic acid $(50$ $\mu \mathrm{M})$ and $\left[1-{ }^{14} \mathrm{C}\right]$ - octanoic acid $(50 \mu \mathrm{M})$ were performed under the same conditions as described above for SVA, except that the mixtures contained $0.025 \mathrm{mg} / \mathrm{mL}$ of mouse liver subcellular fractions for palmitic acid and $0.125 \mathrm{mg} / \mathrm{mL}$ for octanoic acid and were incubated for $5 \mathrm{~min}$. Values represent means \pm SD from triplicate incubations. 
Table $1 .{ }^{1} \mathrm{H}$ and ${ }^{13} \mathrm{C}$ chemical shifts of SVA, simvastatin and CoASH-dependent SVA metabolites.

All chemical shifts are referenced to $\mathrm{CD}_{2} \mathrm{HOD}$ peak set at $\delta_{\mathrm{H}} 3.30 / \delta_{\mathrm{C}} 47.9 \mathrm{ppm}$. The decalin ring and the dimethyl butanoic acid moieties are unchanged in all structures and their chemical shifts in all structures were practically identical to previously published data on SVA (Prueksaritanont et al., 2001).

\begin{tabular}{|c|c|c|c|c|c|c|c|c|c|c|}
\hline \multirow[t]{2}{*}{$\operatorname{Label}^{a}$} & \multicolumn{2}{|c|}{$\mathrm{SVA}_{2} \mathrm{CoASH}^{b}$} & \multicolumn{2}{|c|}{ SVA-CoA } & \multicolumn{2}{|c|}{ P1-CoA } & \multicolumn{2}{|c|}{ simvastatin } & \multicolumn{2}{|c|}{ M1 } \\
\hline & ${ }^{1} \mathrm{H}$ & ${ }^{13} \mathrm{C}$ & ${ }^{1} \mathrm{H}$ & ${ }^{13} \mathrm{C}$ & ${ }^{1} \mathrm{H}$ & ${ }^{13} \mathrm{C}$ & ${ }^{1} \mathrm{H}$ & ${ }^{13} \mathrm{C}$ & ${ }^{1} \mathrm{H}$ & ${ }^{13} \mathrm{C}$ \\
\hline 2 & - & 178.5 & - & 197.9 & - & 190.0 & - & 172.0 & - & 172.3 \\
\hline 3 & $2.28,2.39$ & 43.9 & $2.71,2.77$ & 50.8 & 6.22 & 129.9 & $2.52,2.71$ & 37.7 & $2.35,2.85$ & 38.7 \\
\hline 4 & 4.08 & 67.8 & 4.21 & 66.8 & 6.90 & 142.6 & 4.24 & 61.9 & 4.19 & 62.8 \\
\hline 5 & 1.55 & 43.8 & 1.60 & 43.4 & 2.35 & 40.0 & $1.74,1.91$ & 35.2 & $1.49,2.24$ & 37.5 \\
\hline 6 & 3.69 & 70.3 & 3.63 & 69.9 & 3.57 & 71.0 & 4.61 & 76.7 & 4.19 & 77.8 \\
\hline 7 & $1.27,1.54$ & 34.4 & $1.17,1.54$ & 32.9 & $1.17,1.55$ & 34.1 & $1.35,1.82$ & 32.7 & $1.36,1.85$ & 32.6 \\
\hline 8 & 1.40 & 24.5 & 1.34 & 24.4 & 1.37 & 24.7 & $1.39,1.49$ & 23.9 & $1.41,1.49$ & 24.6 \\
\hline$a^{\prime}$ & 2.58 & 23.1 & 3.00 & 27.9 & 3.04 & 27.6 & & & & \\
\hline$b^{\prime}$ & 3.31 & 42.5 & 3.31 & 38.6 & 3.32 & 38.8 & & & & \\
\hline$c^{\prime}$ & - & 172.8 & - & 172.7 & - & 172.7 & & & & \\
\hline d' & 2.45 & 35.3 & 2.41 & 35.2 & 2.43 & 35.3 & & & & \\
\hline $\mathrm{e}^{\prime}$ & 3.46 & 35.1 & 3.45 & 35.1 & 3.46 & 35.1 & & & & \\
\hline$f^{\prime}$ & - & 174.3 & - & 174.4 & - & 174.4 & & & & \\
\hline g' & 4.05 & 73.9 & 4.06 & 73.5 & 4.08 & 73.5 & & & & \\
\hline h' & - & 38.8 & - & 38.7 & - & 38.7 & & & & \\
\hline h'-Me & $0.82,1.02$ & $18.3,20.8$ & $0.83,1.05$ & $18.1,21.0$ & $0.82,1.05$ & $18.1,20.9$ & & & & \\
\hline i’ & $3.59,3.96$ & 71.8 & $3.56,3.98$ & 71.8 & $3.57,3.99$ & 71.7 & & & & \\
\hline $\mathrm{R} 1$ & 6.13 & 87.4 & 6.12 & 87.1 & 6.14 & 87.1 & & & & \\
\hline $\mathrm{R} 2, \mathrm{R} 4$ & $4.76,4.92$ & $74.1,74.6$ & $4.79,4.86$ & $74.1,74.4$ & $4.79,4.92$ & $74.1,74.5$ & & & & \\
\hline R3 & 4.52 & 83.3 & 4.50 & 83.4 & 4.50 & 83.4 & & & & \\
\hline R5 & 4.28 & 64.9 & 4.24 & 65.1 & $4.23,4.30$ & 64.9 & & & & \\
\hline $\mathrm{A} 2$ & 8.22 & 150.3 & 8.20 & 152.4 & 8.20 & 152.4 & & & & \\
\hline A6 & 8.62 & 140.6 & 8.57 & 139.6 & 8.59 & 139.7 & & & & \\
\hline
\end{tabular}

\footnotetext{
${ }^{a}$ Labels are shown in Figure 1
}

${ }^{b}$ Chemical shifts of a' to i', R1 to R5 and A2 \& A6 in this column correspond to the CoASH standard in $90 \% \mathrm{CD}_{3} \mathrm{OD} / 10 \% \mathrm{D}_{2} \mathrm{O}$. 


\section{DMD \#6650}

Table 2. Observed and calculated $\mathrm{m} / \mathrm{z}$ values of the most abundant fragment ions in the MS/MS spectra of simvastatin standard (A) and M1 (B).

The calculated values and formulas are based on the fragmentation assigned in Figure 5. The MS/MS spectra were obtained using a Micromass Q-TOF mass spectrometer (see text for details).

A. simvastatin standard

\begin{tabular}{lcccc}
\hline Elemental Composition & Observed $\mathrm{m} / z$ & Calculated $\mathrm{m} / z$ & Deviation $(\mathrm{mDa})$ & Deviation (ppm) \\
\hline $\mathrm{C}_{13} \mathrm{H}_{17}{ }^{+}$ & 173.1328 & 173.1325 & -0.3 & -1.7 \\
$\mathrm{C}_{15} \mathrm{H}_{19}{ }^{+}$ & 199.1486 & 199.1481 & -0.5 & -2.5 \\
$\mathrm{C}_{17} \mathrm{H}_{21}{ }^{+}$ & 225.1638 & 225.1638 & 0.0 & 0.0 \\
$\mathrm{C}_{17} \mathrm{H}_{23} \mathrm{O}^{+}$ & 243.1744 & 243.1743 & -0.1 & -0.4 \\
$\mathrm{C}_{19} \mathrm{H}_{23} \mathrm{O}^{+}$ & 267.1762 & 267.1743 & -1.9 & -7.1 \\
$\mathrm{C}_{19} \mathrm{H}_{25} \mathrm{O}_{2}^{+}$ & 285.1856 & 285.1849 & -0.7 & -2.5 \\
$\mathrm{C}_{19} \mathrm{H}_{27} \mathrm{O}_{3}{ }^{+}$ & 303.1964 & 303.1955 & -0.9 & -3.0 \\
\hline
\end{tabular}

B. M1

\begin{tabular}{lcccc}
\hline Elemental Composition & Observed $m / z$ & Calculated $m / z$ & Deviation (mDa) & Deviation (ppm) \\
\hline $\mathrm{C}_{13} \mathrm{H}_{17}{ }^{+}$ & 173.1326 & 173.1325 & -0.1 & -0.6 \\
$\mathrm{C}_{15} \mathrm{H}_{19}{ }^{+}$ & 199.1490 & 199.1481 & -0.9 & -4.5 \\
$\mathrm{C}_{17} \mathrm{H}_{21}{ }^{+}$ & 225.1655 & 225.1638 & -1.7 & -7.6 \\
$\mathrm{C}_{17} \mathrm{H}_{23} \mathrm{O}^{+}$ & 243.1744 & 243.1743 & -0.1 & -0.4 \\
$\mathrm{C}_{19} \mathrm{H}_{23} \mathrm{O}^{+}$ & 267.1742 & 267.1743 & 0.1 & 0.4 \\
$\mathrm{C}_{19} \mathrm{H}_{25} \mathrm{O}_{2}^{+}$ & 285.1855 & 285.1849 & -0.6 & 2.1 \\
$\mathrm{C}_{19} \mathrm{H}_{27} \mathrm{O}_{3}^{+}$ & 303.1945 & 303.1955 & 1.0 & 3.3 \\
\hline
\end{tabular}


DMD \#6650

Table 3. Marker enzyme activities in mouse liver subcellular fractions.

\begin{tabular}{|c|c|c|c|c|c|c|c|}
\hline \multirow[t]{2}{*}{ Marker enzymes } & \multirow{2}{*}{$\begin{array}{c}\text { Specific activity } \\
\text { of } \mathrm{S} 0.6^{a}\end{array}$} & \multicolumn{6}{|c|}{ Specific activity relative to S0.6 } \\
\hline & & S3 & MT-H & $\mathrm{S} 27$ & MT-L & cytosol & microsomes \\
\hline Catalase & $167 \pm 13$ & $0.83 \pm 0.01$ & $1.26 \pm 0.08$ & $0.52 \pm 0.04$ & $2.79 \pm 0.10$ & $0.68 \pm 0.04$ & $0.41 \pm 0.01$ \\
\hline Cytochrome c oxidase & $217 \pm 3$ & $0.72 \pm 0.04$ & $3.21 \pm 0.23$ & $0.29 \pm 0.07$ & $2.89 \pm 0.52$ & $0.02 \pm 0.03$ & $0.93 \pm 0.16$ \\
\hline Lactate dehydrogenase & $11.1 \pm 1.0$ & $0.99 \pm 0.11$ & $0.18 \pm 0.11$ & $1.08 \pm 0.08$ & $0.06 \pm 0.003$ & $1.63 \pm 0.18$ & $0.08 \pm 0.004$ \\
\hline Cytochrome $\mathrm{c}$ reductase & $9.7 \pm 0.6$ & $0.94 \pm 0.10$ & $0.03 \pm 0.01$ & $1.26 \pm 0.05$ & $0.10 \pm 0.02$ & $0.24 \pm 0.01$ & $3.56 \pm 0.19$ \\
\hline
\end{tabular}

${ }^{a}$ Units for catalase and lactate dehydrogenase are $\mu \mathrm{mol} / \mathrm{min} / \mathrm{mg}$ protein; units for cytochrome c oxidase and cytochrome $\mathrm{c}$ reductase are $\mathrm{nmol} / \mathrm{min} / \mathrm{mg}$ protein.

Values represent means \pm SD from triplicate incubations. 


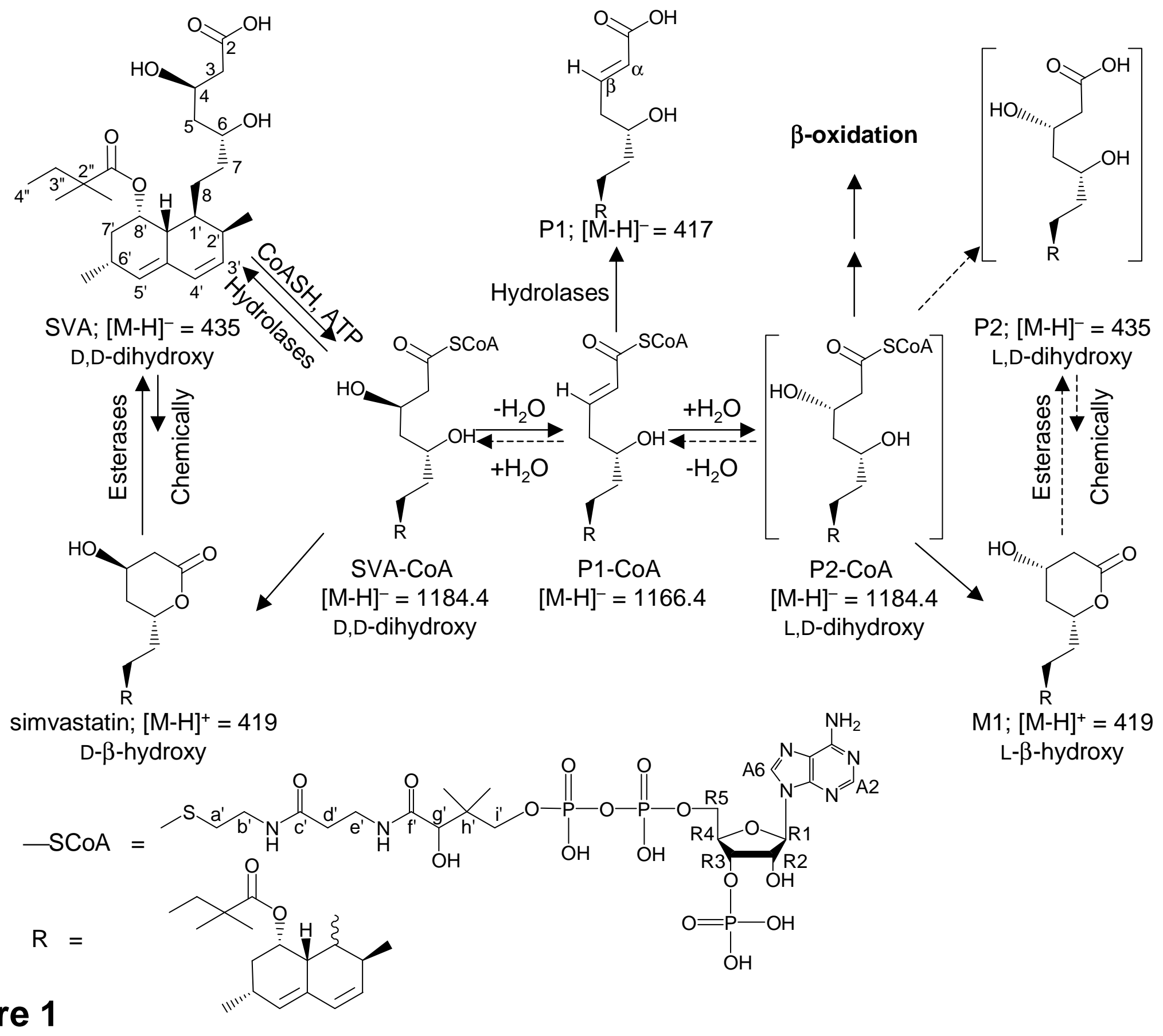




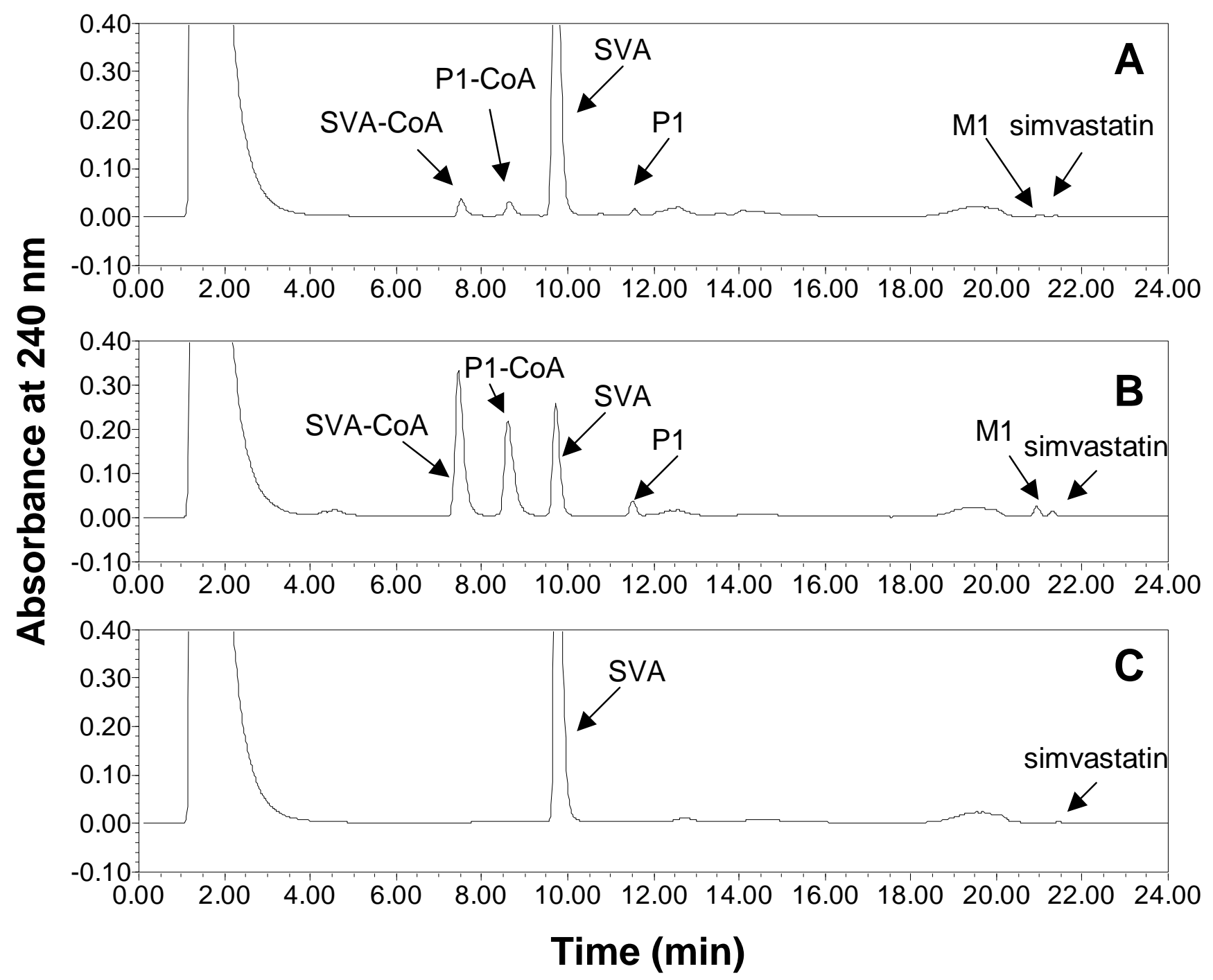

Figure 2 
Figure 3

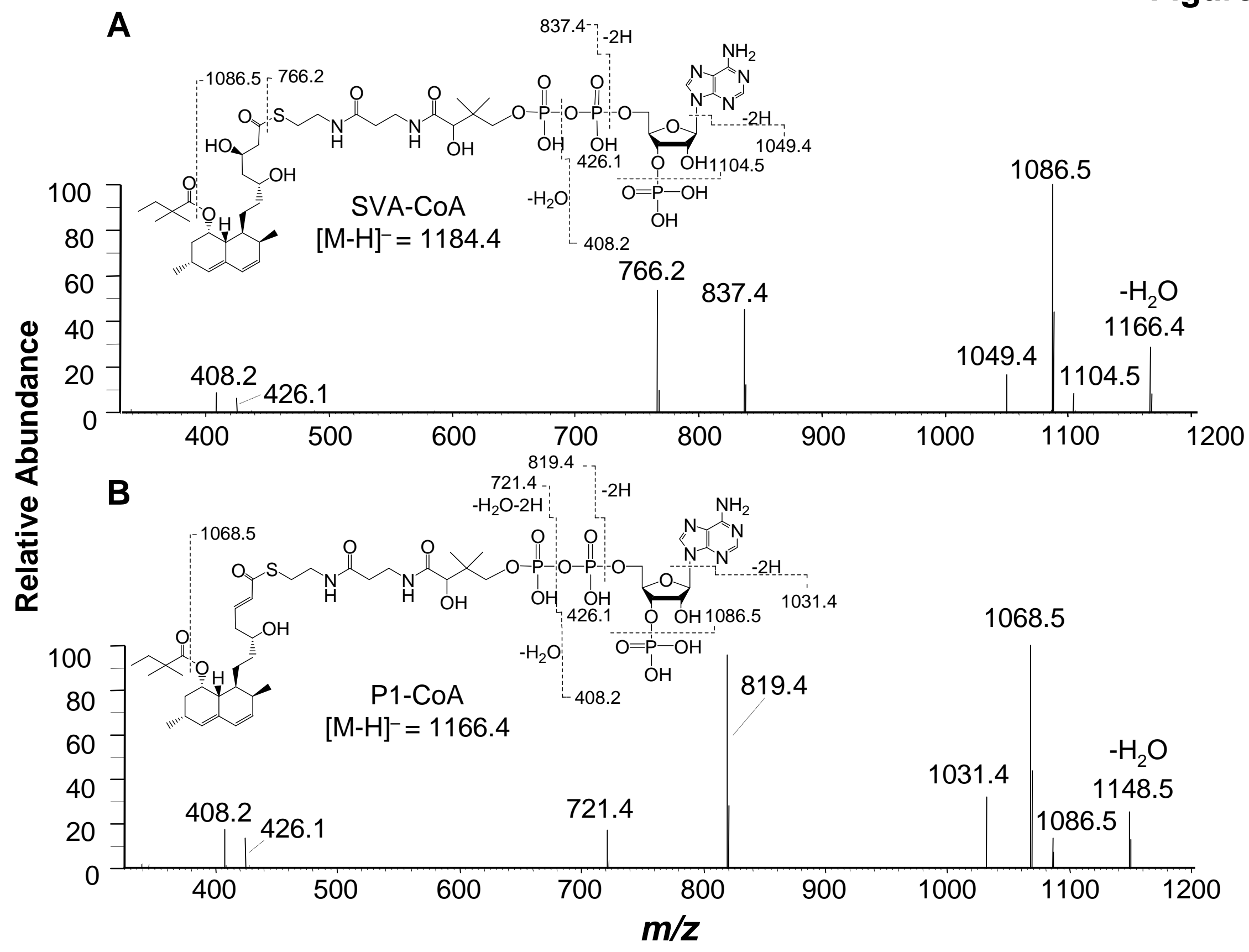


Figure 4
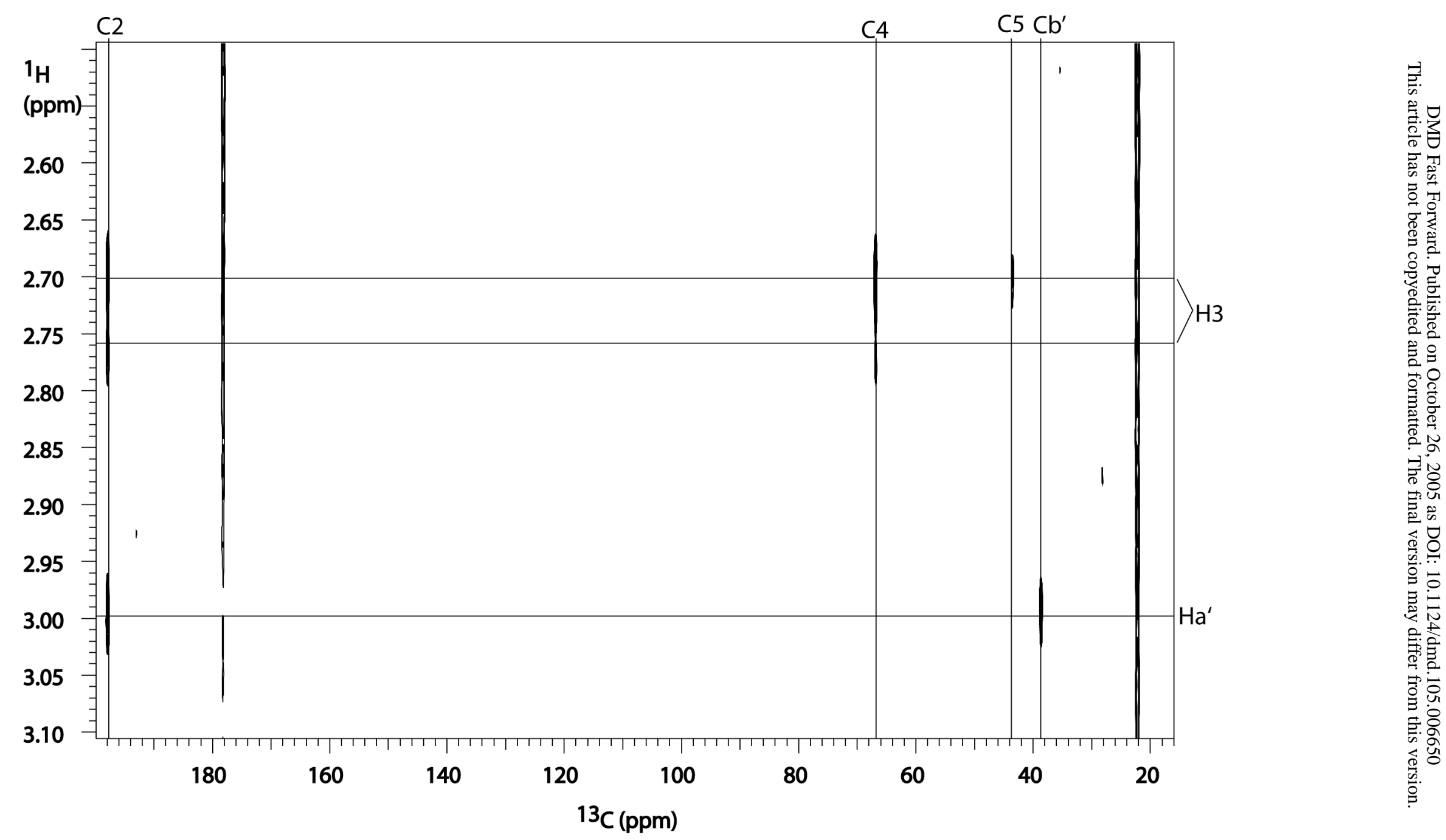


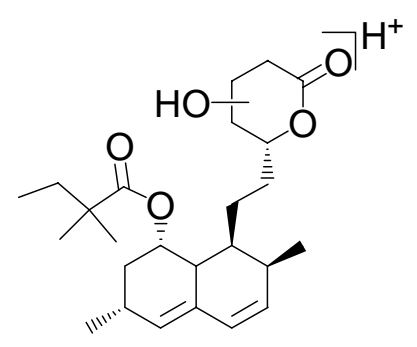

$$
\mathrm{C}_{25} \mathrm{H}_{39} \mathrm{O}_{5}^{+}
$$

Exact Mass: 419.2792

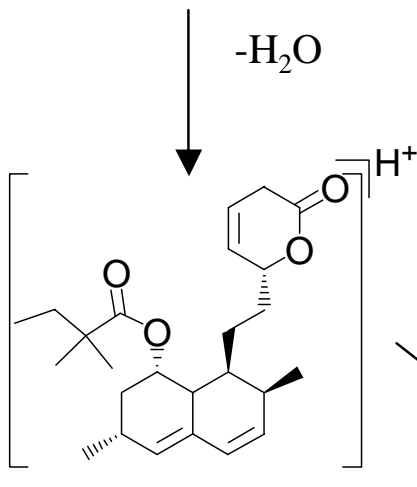

$$
\mathrm{C}_{25} \mathrm{H}_{37} \mathrm{O}_{4}^{+}
$$

Exact Mass: 401.2686

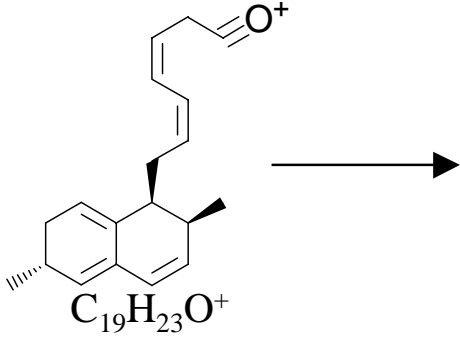

Exact Mass: 267.1743
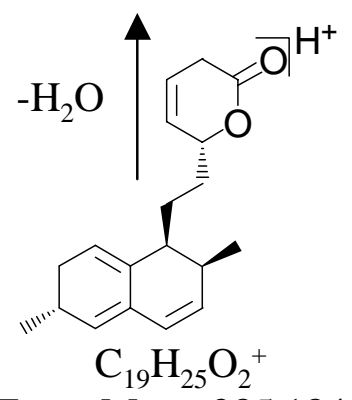

Exact Mass: 285.1849

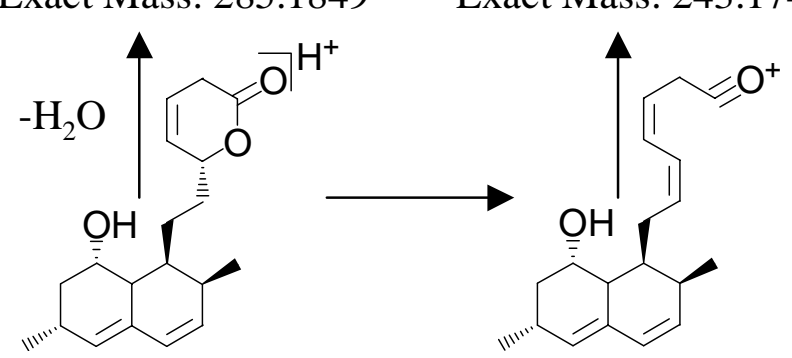

$$
\mathrm{C}_{19} \mathrm{H}_{27} \mathrm{O}_{3}^{+}
$$

Exact Mass: 225.1638

Exact Mass: 243.1743

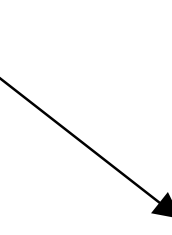$$
\mathrm{C}_{19} \mathrm{H}_{25} \mathrm{O}_{2}^{+}
$$
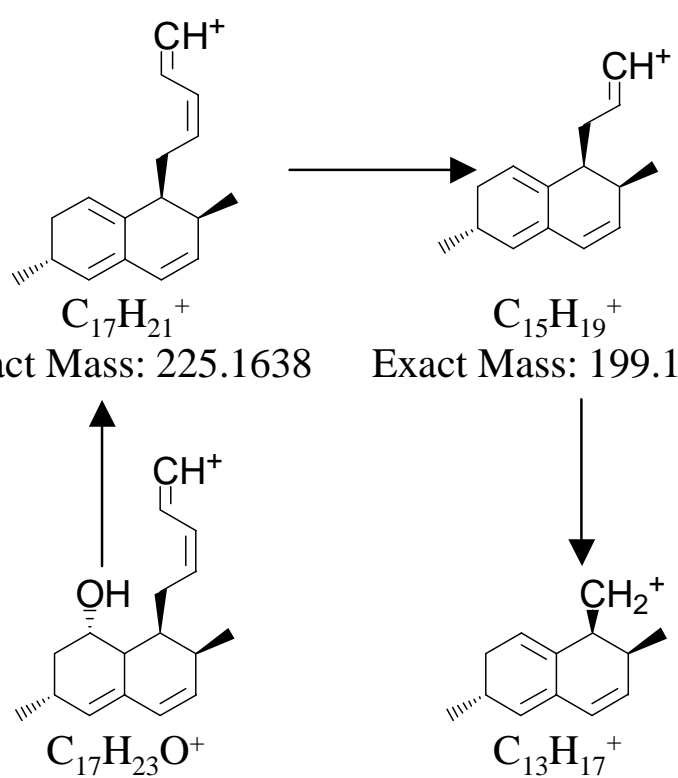

Exact Mass: 199.1481

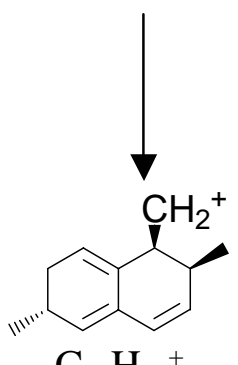

$$
\mathrm{C}_{13} \mathrm{H}_{17}^{+}
$$

Exact Mass: 173.1325

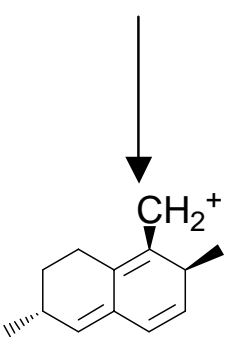

$\mathrm{C}_{13} \mathrm{H}_{17}^{+}$

Exact Mass: 173.1325

Figure 5 


\section{Figure 6}

A.

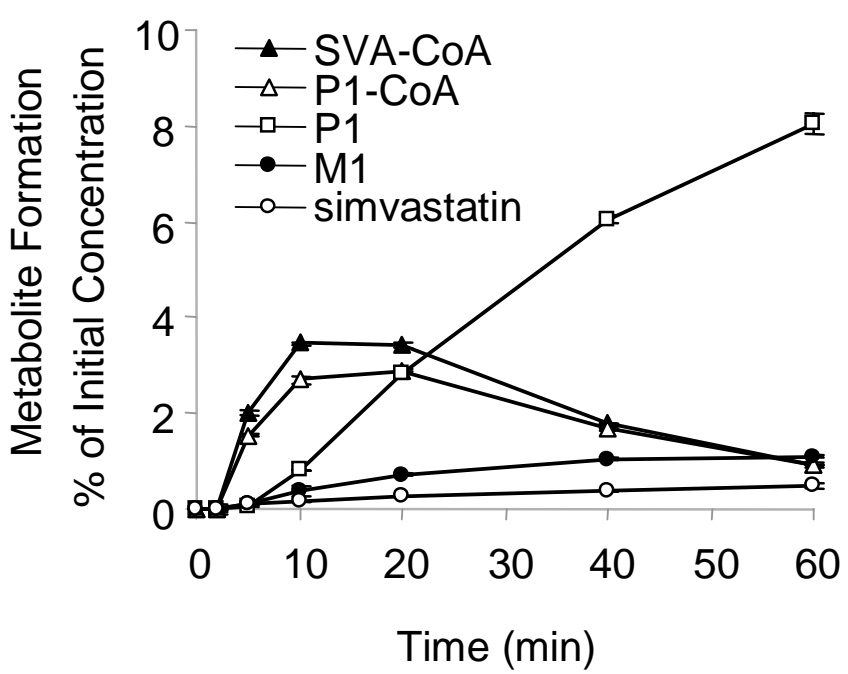

B.

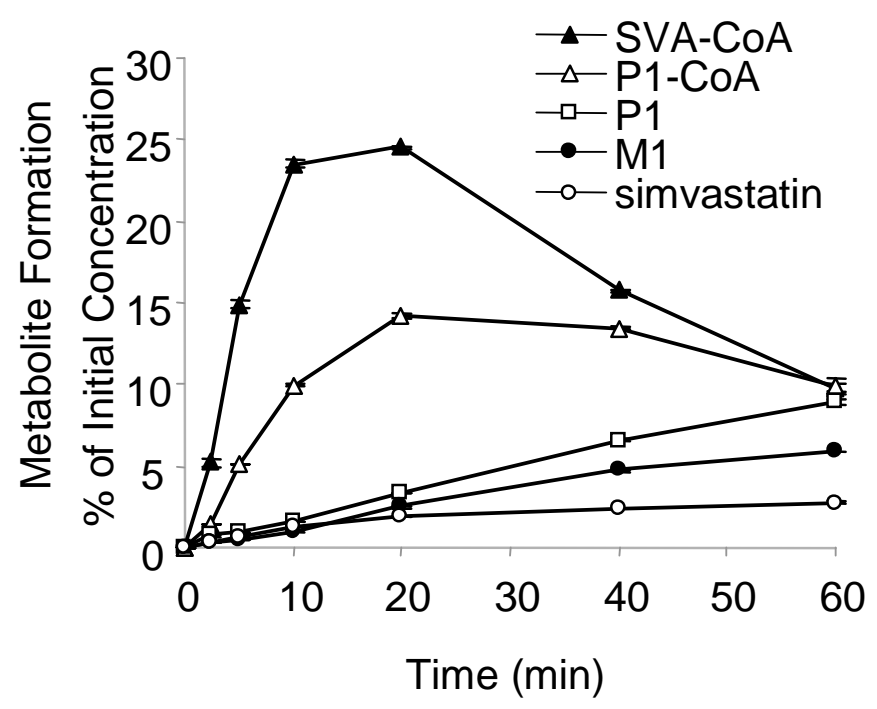


Figure 7

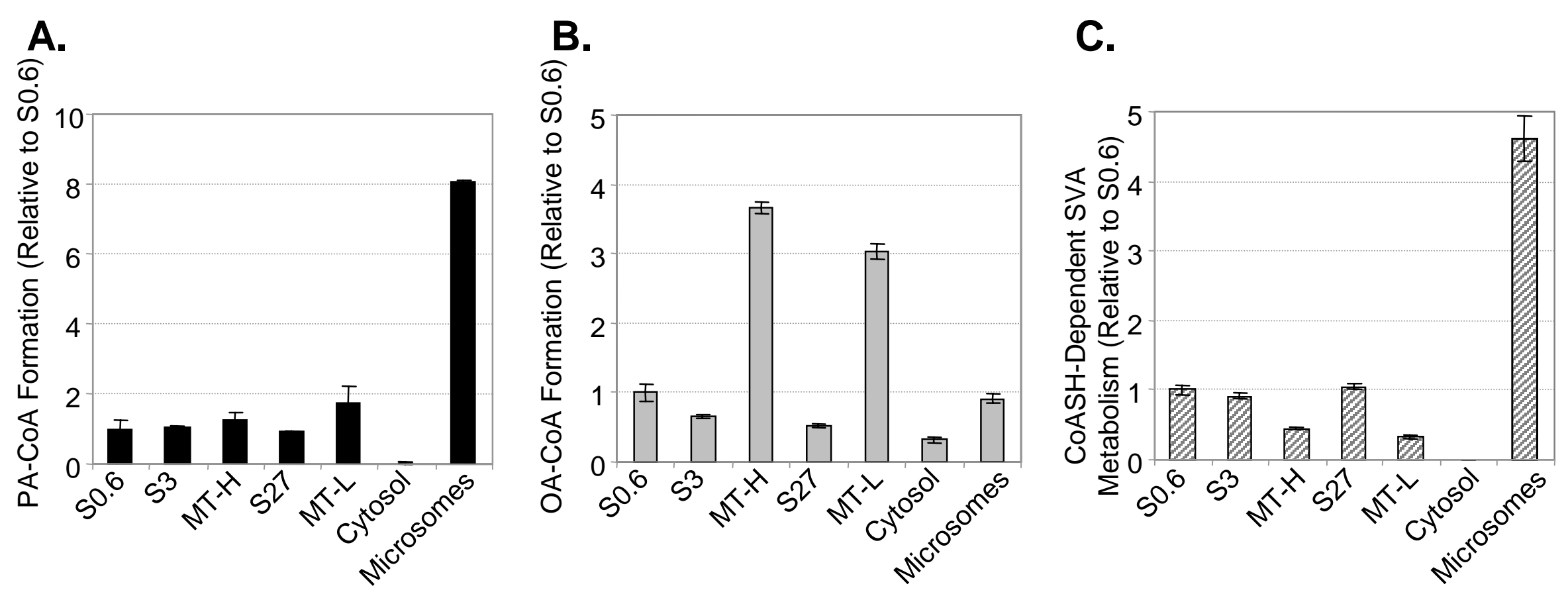

